\title{
SARS-COV-2 AMONG FRONT LINE HEALTH CARE WORKERS
}

\author{
Syed Waqar Abbas, Shoaib Ahmed, Syeda Fatimah Zareen, Sadia Fatima, Nusrat Rehan, Wajid Hussain* \\ Combined Military Hospital/National University of Medical Sciences (NUMS) Rawalpindi Pakistan, *Armed Forces Institute of \\ Pathology/National University of Medical Sciences (NUMS) Rawalpindi Pakistan
}

\begin{abstract}
Objective: To determine clinical presentations and source among severe acute respiratory syndrome corona virus 2 positive health care workers of tertiary care hospital.

Study Design: Cross-sectional study.

Place and Duration of Study: Combined Military Hospital Rawalpindi, from Mar to Jun 2020.

Methodology: A cross sectional study was conducted by using a questionnaire for risk assessment regarding exposure to Corona Virus Disease 2019 (COVID-19) virus and practices of participants. Health care workers who were exposed or had fever, cough, shortness of breath, were asked to undergo semi-quantitative real-time reverse transcriptase-polymerase chain reaction test for Severe Acute Respiratory Syndrome Corona Virus 2 on nasopharyngeal and oro-pharyngeal swabs.

Results: Out of the 92 health care workers who tested positive, 80/92 (86.95\%) were males, 12/92 (13.04\%) females. Asymptomatic cases were 53/92 (57.60\%) and 39/92 (42.40\%) were symptomatic, $10 / 92(10.8 \%)$ of the participants were doctors, 06/92 (6.52\%) nurses, 36/92 (39.14\%) paramedics and 40/92 (43.47\%) were hospital auxiliary staff. Among symptomatic cases, 28/39 (71.79\%) developed fever with myalgia, 22/39 fever alone, $23 / 39(58.97 \%)$ headache, $13 / 39(33 \%)$ sore throat, $15 / 39(38 \%)$ runny nose, $11 / 39(28.20 \%)$ mild shortness of breath on exertion, $7 / 39$ (17.94\%) diarrhea and 5/39 (12.82\%) experienced loss of taste.

Conclusion: As the Corona Virus Disease 2019 pandemic continues, chances of health care workers getting infected are high so it is critical to improve the knowledge of Health care workers. Educational interventions and further studies are warranted in this regard.
\end{abstract}

Keywords: Corona Virus Disease 2019, Health care workers, Nasopharyngeal, Oropharyngeal swab, Polymerase chain reaction.

This is an Open Access article distributed under the terms of the Creative Commons Attribution License (http://creativecommons.org/licenses/by/4.0), which permits unrestricted use, distribution, and reproduction in any medium, provided the original work is properly cited.

\section{INTRODUCTION}

The Pandemic of coronavirus (COVID-19) was declared a Public Health Emergency (PHE) by World Health Organization (WHO) ${ }^{1}$ in late January 2020. More than 200 countries around the world have been affected by virus so far. As of 23rd June 2020, there were 9,063,264 confirmed cases and 471, 681 deaths worldwide. In Pakistan, on the same date, there were reportedly total 185,034 confirmed cases and 3,695 deaths ${ }^{2}$. COVID-19 is caused by a strain of coronavirus (SARS-COV-2) and the pandemic started in Dec 2019, from Chinese province of Wuhan. SARS$\mathrm{CoV}-2$ infection is actually zoonosis that can

Correspondence: Dr Syed Waqar Abbas, Classified Medical Specialist, Combined Military Hospital, Rawalpindi Pakistan

Received: 13 Jul 2020; revised received: 13 Aug 2020; accepted: 17 Aug 2020 spread through animal-to-human and human-tohuman contact ${ }^{3}$. The most agreed upon mode of human-to-human transmission is through respiratory droplets produced during coughing, sneezing and talking. Its airborne spread has not been yet confirmed; so far air sampling has found virus RNA in some studies ${ }^{4}$ but not in others ${ }^{5}$. In addition to respiratory droplets, direct contact (shaking hands) with positive individual or an infected surface followed by touching the mouth, nose or eyes is also an important mode of transmission ${ }^{4}$. The incubation period of COVID-19 is considered to be 5-7 days. The total duration of illness from appearance of symptoms to recovery or fatal outcome varies from 6 to 41 days with a median of 14 days $^{6}$. Common clinical presentations include fever, cough, difficulty in breathing, headache, loss of sense of smelland taste, aches 
and pain, loose stools and flu like symptoms and fatiguability ${ }^{6}$.

Following the initiation of emergency responses, healthcare workers did not get adequate time to undergo planned training and practices. Professional supervision and guidance, as well as monitoring mechanism were evolving under the impact of frequent changes in health care guidelines and relevant WHO instructions. This scenario in fact aggravated the dangers of infection in healthcare workers (HCWs) ${ }^{1}$. It is therefore, need of the hour that health systems and health care organizations develop infrastructures and attain resources to support physicians, nurses and ancillary members.

The awareness regarding importance of personal protection, religious use of sufficient PPE, and proper preparedness and response would be of great help in lowering the risk of infection among healthcare workers ${ }^{2}$. A lot of debate is going on over various forums as well as by public health authorities, about use of face masks and eye protection, especially the use of face masks for the general population7. Governments in many countries have employed strict isolation and lock down policies in an attempt to avoid the potentially disastrous consequences ${ }^{8}$. The paradox, however, is that whilst most people are supposed to remain confined to homes during the pandemic, the converse is true for health-care workers 9,10 . The nature of their job, along with prolonged duty hours (due to increased number of infected fellow workers) make them prone to infection. Furthermore, the shortage of appropriate PPE consequent to a sudden massive rise in demand, has led to more hurdles in achieving full protection against infection.

It is of utmost importance to propagate information relevant to COVID-19 and associated health matters at pertinent levels, in order to educate and guide our frontline warriors, the Health Care Workers, in a simple and easy to learn manner. Moreover, it is equally important to stop spread of fake and wrong information that is readily available on social media and few unauthentic web sites ${ }^{13}$.

\section{METHODOLOGY}

A cross sectional study carried out at Combined Military Hospital Rawalpindi on health care workers (HCWs), for three months duration from 22nd March 2020 to 22nd June 2020. The ethics and review committee reviewed and approved the study, reference number 92/06/20(29). Total of 92 HCWs Infected with COVID-19 including doctors, nurses, medical assistants, paramedics, were included in the study. There were no particular exclusion criteria. Participation was voluntary and a consent statement for all participants was included in the questionnaire and they were given the right to confidentiality. Data was collected that comprised predefined responses including the demographic and risk assessment sections. The first section of the questionnaire consisted of closed ended questions about the demographic details such as age, gender, designation and type of workplace. Section two had questions focused on exposure of health care Workers to COVID-19 virus. These included date of direct or indirect exposure with COVID19 positive patient, PCR sample date, distance from the patient, duration of exposure, wearing of PPE details, use of surgical gloves and N95 Mask and development of symptoms with their duration. The questionnaire was evaluated for validity by colleagues in infectious disease, internal medicine and intensive care. The questionnaire responses were recorded on phone and on direct session in order to assess the risk factors amongst infected health care professionals Data was analyzed by using SPSS version 22.0. Mean \pm SD were calculated for continuous variable like age. Frequency and percentages were obtained for qualitative variables like gender. Descriptive statistics were used to describe the result.

\section{RESULTS}

A total of 92 HCWs were interviewed and completed the given questionnaire. Out of the 92 HCWs, 80/92 (86.95\%) were males and 12/92 $(13.04 \%)$ females. Mean Age was $35 \pm 15$ years as 
shown in table-I. Among the included participants 10/92 (10.86\%) were doctors, 06/92 (6.52\%) nurses, 36/92 (39.14\%) paramedics and 40/92 $(43.47 \%)$ were hospital auxiliary staff like Clerks,

Table-I: Ages of the SARS CoV-2 positive health care workers.

\begin{tabular}{l|c}
\hline Age in Years & n (\%) \\
\hline $0-30$ & $49 / 92(53.26 \%)$ \\
\hline $31-40$ & $25 / 92(27.16 \%)$ \\
\hline $40-50$ & $16 / 92(17.39 \%)$ \\
\hline$>50$ & $02 / 92(2.17 \%)$ \\
\hline
\end{tabular}

Ayas, Sanitary workers, Drivers and others as shown in fig-1. Total 58/92 (63.04\%) health Care workers had direct contact with COVID-19

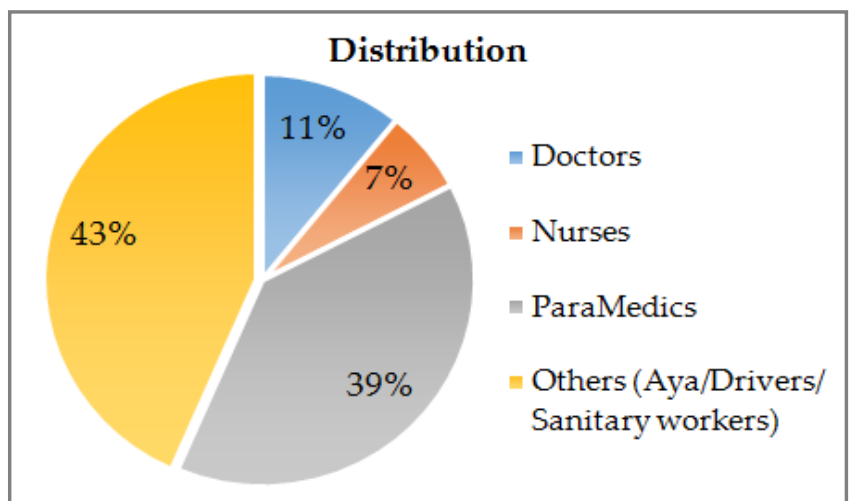

Figure-1: Distribution of COVID-19 positive health care workers.

patients and in 34/92 (36.95\%), source of infection was unknown or through indirect contact with positive cases as shown in table-II. 53/92 of $38.0^{\circ} \mathrm{C}$ or higher), $22 / 39(56 \%)$ had fever alone, 23/39 (58.97\%) headache, 13/39 (33\%) sore throat, $15 / 39$ (38\%) runny nose, $11 / 39$ (28.20\%) mild shortness of breath especially on exertion or climbing stairs, 7/39 (17.94\%) diarrhea and 5/39 $(12.82 \%)$ complained of loss of taste as shown in fig-2. Three HCWs disclosed that they already had known allergy and occasional symptoms before infection. Health care workers especially consultants and trainee doctors had generally taken adequate protective measures and followed SOPs.

\section{DISCUSSION}

Since its appearance in December 2019, COVID-19 pandemic has rapidly gripped the world with devastating consequences ${ }^{11}$. Currently, this infection is probably the sole health issue of concern all over the world. It is the most com-monly discussed issue among people from all walks of life now a days ${ }^{12}$. At the same time, it is a matter of great anxiety and apprehension among health care workers as well as the patients and their families. One may hope that matters will improve with improved practices over the course of next few months.

Our study was designed to determine the sources of exposure in SARS-COV-2 infected HCWs along with symptomatology. So far, Infected HCWs at our hospital experienced milder disease as compared to the clinical presentation

Table-II: COVID-19 virus exposure of health care workers (HCW).

\begin{tabular}{c|l|c|c}
\hline S. No & Important points & Yes, n (\%) & No, n (\%) \\
\hline 1 & $\begin{array}{l}\text { Did you have direct contact with the environment where } \\
\text { COVID patients was cared for? }\end{array}$ & $58 / 92(63.04 \%)$ & $34 / 92(36.95 \%)$ \\
\hline 2 & $\begin{array}{l}\text { During the health care interaction with a COVID-19 } \\
\text { patients, did you wear Personal Protective Equipment PPE) }\end{array}$ & $52 / 92(56.52 \%)$ & $40 / 92(43.48 \%)$ \\
\hline 3 & Did you wear proper N95 mask, gloves and apron? & $25 / 92(27.17 \%)$ & $67 / 92(72.82 \%)$ \\
\hline
\end{tabular}

(57.6\%) were asymptomatic at the start, this implies that these individuals were tested because of history of contact with other positive healthcare workers in resting areas or with patients. Those who were symptomatic experienced mild disease. Out of 39 symptomatic cases 28/39 (71.79\%) developed fever with myalgia (body temperature and outcomes reported for other hospitalized patients, but fatality reports have been documented in a significant number of health care individuals in various other setups ${ }^{14}$.

In our study, 58/92 (63.04\%) HCWs had direct contact with COVID-19 patients and in $34 / 92(36.95 \%)$, source of infection remained un- 
known. It is very difficult to exactly identify the mode of transmission of COVID-19 within the hospital through routine data, since the hospitals are subjected to numerous external sources of infection. Among frontline fighters (Health Care Workers), reuse of Personnel Protective Equipment or inadequate protection rendered by PPE, indicating either inadequate supply or poor quality PPE material, was the factor responsible for a subsequent $31-46 \%$ increased risk of COVID-19.

Table-III: Symptoms of patients.

\begin{tabular}{l|c}
\hline Symptoms & Frequency, n (\%) \\
\hline Fever \& Myalgia & $28 / 39(71.74 \%)$ \\
\hline Fever & $22 / 39(56.41 \%)$ \\
\hline Shortness of breath & $11 / 39(28.20 \%)$ \\
\hline Headache & $23 / 39(58.97 \%)$ \\
\hline Runny Nose & $15 / 39(38.46 \%)$ \\
\hline Sore Throat & $13 / 39(33.33 \%)$ \\
\hline Diarrhea & $07 / 39(17.94 \%)$ \\
\hline Loss of Taste & $05 / 39(12.82 \%)$ \\
\hline
\end{tabular}

Poor donning and doffing techniques (steps) resulting in self- contamination, in addition to worn out dresses due to extended use and low quality material of PPE, pose an even greater risk of infection in HCWs. Even with use of PPE, HCWs especially those who were directly involved in care of COVID-19 patients, remained at elevated risk for catching the infection. In our study, large proportion of the HCWs 53/92 (57.6\%) was asymptomatic, who likely had contracted infection through secondary or tertiary hospital-acquired source which could have been prevented by timely identifying and isolating the SARS-COV-2 positive cases for an appropriate duration. An important finding was the presence of asymptomatic infection in $22 \mathrm{HCWs}$ who shared accommodation facilities at unit lines which further emphasizes the value of early detection and isolation as well as social distancing. Similarly 12 HCWs were resident of Tench Bhata, an over populated area of Rawalpindi where many COVID-19 cases were documented. There observations point towards the possibility of HCWs catching infection from around their residential areas and surroundings. Obviously PPE is suppo- sed to be used while being on duty and the chances of getting infection from outside the hospital do increase if area of residence is known to be contaminated and carelessness is exercised in use of face mask and social distancing. We suggest that with limited testing capacity, we should give high priority to such asymptomatic cases to prevent uncontrolled staff to staff or staff to patient transmission. We also recommended to increase testing of these asymp-tomatic contacts among HCWs when testing capacity increases.

In our study, the most common categories of HCWs found COVID-19 positive were paramedics 36/92 (39.14\%) and hospital auxiliary staff 40/92 (43.47\%) like Clerks, Ayas, Sanitary workers \& Drivers. This indicates inadequate and compromised personal protective measures practiced by these categories. Hospital lower staff was also reported as most commonly affected HCWs category. To give adequate knowledge and understanding of COVID-19 to health care workers of our hospital, a series of regular training sessions have been conducted at all departments for all tiers of hospital staff. These were aimed at apprising everybody on personal safety and prevention, awareness about personnel protective equipment (PPE) and their use (donning and doffing), nosocomial transmission, hand hygiene and sanitization ${ }^{15}$. During this COVID-19 pandemic, implementations of infection prevention control (IPC) measures in health care and hospital settings, predominantly the use of personal protection equipment (PPE) by healthcare workers, became the most important issues ${ }^{16}$. Most of these sessions have been interactive and involve hands-on training. Lower staff are also encouraged to actively participate and freely ask questions. Clinical meetings are regularly conducted by senior consultants in Medicine to analyze the rapidly changing COVID situation and evolving challenges. This helps in reaching prompt decisions with the input of all stakeholders.

A meta-analysis of 64 studies with record taken from WHO database reported that a substantial number of Health care workers may be at risk of getting infected by coronavirus especially 
when protective measures are inadequate. The same study further reported that clinical symptoms in COVID-19 infected HCWs were milder than other patient populations, results similar to our study. Improper protective measures especially carelessness in use of masks followed by eye protection, hand hygiene etc were found to be the main risk factors for transmission of disease in HCWs, as was also observed in our study. It was also observed that training in use of protective measures may prove helpful in reducing the disease transmission ${ }^{19}$.

Another study done at Italy found that common symptoms in SARS-COV-2 infected HCWs were fever, myalgias, anosmia, dysgeusia and mild shortness of breath with one-third being asymptomatic. This is slightly different from our study where more than fifty percent infected HCWs were asymptomatic ${ }^{20}$.

Till the development of effective and an efficacious vaccine, the chances of second waves of COVID-19 are very high ${ }^{17}$. The reason behind is that the social distancing rules and duration of isolation, the most important factors ${ }^{18}$ are relaxed to some extent now as the country is turning towards smart lock-down. The hospital infection control policies need to be strengthened in order to limit the hospital transmission of infection between the HCWs and patients. Recently serological testing is widely available in big cities which may help in identifying the cases and their infectivity. Our data suggest that the roll-out of screening programs to include asymptomatic as well as symptomatic patient-facing staff should be a national and international priority.

\section{RECOMMENDATION}

The policy of social distancing needs to be strictly implemented in all areas of the hospital. Wearing of proper masks (surgical mask, not cloth made) should be considered mandatory for every individual visiting the hospital. Proper N95 masks to be provided to all consultants, residents, house officers, nurses and paramedical staff, who are directly involved in patient care. Importance and positive effects of Hand washing and saniti- zation should be highlighted at all possible levels. Telemedicine should be strengthened to regulate the number of patients presenting at OPD clinics. There should be an upper limit of the number of patients. Dispatch of medicine through courier also needs to be encouraged to reduce patient congestion at pharmacy. Continuous daily emphasis and short training sessions on proper wearing of face mask, importance of physical distancing (inside \& outside the hospital), hand washing and use of sanitizer is particularly essential for paramedical and auxiliary staff. A database needs to be maintained to include details of every patient diagnosed COVID-19 positive to facilitate understanding of disease epidemiology.

\section{CONCLUSION}

Our study suggests that most of the health care workers have reasonably good knowledge and understanding about COVID-19 but confusion exist because of the daily new emerging situation related to mode of transmission, incubation period, treatment, and vaccination. However, some aspects of practices of health care professionals were found to be deficient including proper maintenance of personal distance because of overcrowding, reduction in duty duration in prime risk areas of exposure, disinfection protocols and the use of proper N-95 mask. We have to decrease the observed risks from health care setting to manage the pandemic and limit the morbidity and mortality related to it especially in frontline care givers.

\section{CONFLICT OF INTEREST}

This study has no conflict of interest to be declared by any author.

\section{REFERENCES}

1. World Health Organization (WHO). Coronavirus disease 2019 (COVID-19): situation report, 144. 2020. Available from: https://www.who.int/docs/default-

source/coronaviruse/ situation-reports/20200612-covid-19sitrep-144.pdf?sfvrsn=66ff9f $4 \mathrm{f} \_4$

2. Government of Pakistan. Coronavirus in Pakistan Confirmed Cases 2020. Available from: http://covid.gov.pk/.

3. Perlman S, Netland J. Coronaviruses post SARS, update on replication and pathogenesis. Nat Rev Microbiol 2009; 7(6): 43950 .

4. Guo ZD, Wang ZY, Zhang SF, Li X, Li L, Li C et al. Aerosol and surface distribution of severe acute respiratory syndrome 
coronavirus 2 in hospital wards, Wuhan, China, 2020. Emerging Infect Dis 2020; 26(7): 1583-91.

5. Li Q, Guan X, Wu P, Wang X, Zhou L. Early transmission dynamics in Wuhan, China, of Novel Coronavirus-Infected Pneumonia. N Engl J Med 2020; 382(13): 1199-207.

6. Rothan HA, Byrareddy SN. The epidemiology and pathogenesis of coronavirus disease COVID-19 outbreak. J Autoimmune 2020; 109(1): 534-40.

7. Feng S, Shen C, Xia N, Song W, Fan M, Cowling BJ. Rational use of face masks in the COVID-19 pandemic. Lancet Respir Med 2020; 8(11): 434-36.

8. Huang C, Wang Y, Li X, Ren L, Zhao J, Hu Y et al. Clinical features of patients infected with 2019 novel coronavirus in Wuhan, China. Lancet 2020; 395(10223): 497-506.

9. The Lancet. COVID-19: protecting health-care workers. Lancet 2020; 395(10240): 922-28.

10. Chan JF, To KK, Tse H, Jin DY, Yuen KY. Interspecies transmission and emergence of novel viruses: lessons from bats and birds. Trends Microbiol 2013; 21(10): 544-55.

11. Yin $Y$, Wunderink RG. MERS, SARS and other coronaviruses as causes of pneumonia. Respirology 2018; 23(2): 130-37.

12. Loeb M, Dafoe N, Mahony J. Surgical mask vs N95 respirator for preventing influenza among health care workers: a randomized trial. J Am Med Assoc 2009; 302(3): 1865-71.

13. $\mathrm{Wu} \mathrm{Z}$. Characteristics of and important lessons from the coronavirus disease 2019 (COVID-19) outbreak in China: summary of a report of 72,314 cases from the Chinese Center for Disease Control and Prevention. J Am Med Assoc 2020; 323(13): 1239-42.
14. Arentz M, Yim E, Klaff L. Characteristics and outcomes of 21 critically ill patients with COVID-19 in Washington State. J Am Med Assoc 2020; 323(16): 1612-14.

15. Cowling BJ, Ali ST, Ng TW. Impact assessment of non-pharmaceutical interventions against coronavirus disease 2019 and influenza in Hong Kong: an observational study. Lancet Public Health 2020; 5(5): e279-88

16. Wang J, Liu F, Tan J, Harbarth S, Pittet D, Zingg W et al. Implementation of infection prevention and control in acute care hospitals in Mainland China - a systematic review. Antimicrob Resist Infect Control 2019; 8(1): 32.

17. Zhu FC, Li YH, Guan XH, Hou LH, Wang WJ, Li JX, et al. Safety, tolerability, and immunogenicity of a recombinant adenovirus type-5 vectored COVID-19 vaccine: a dose-escalation, openlabel, non-randomized, first-in-human trial. Lancet 2020; 395 (10240): 1845-54.

18. Kucharski J, Klepac P, Conlan A, Kissler S, Tang, Fry H et al. Effectiveness of isolation, testing, contact tracing, and physical distancing on reducing transmission of SARS-CoV-2 in different settings: a mathematical modelling study. Lancet Infect Dis 2020; 10(1016): 357-62.

19. Chou R, Dana T, Buckley DI, Selph S, Fu R, Totten AM, et al. Epidemiology of and risk factors for coronavirus infection in health care workers: A Living Rapid Review. Ann Intern Med 2020; 173(2): 120-36.

20. Magnavita N, Tripepi G, Di Prinzio RR. Symptoms in health care workers during the COVID-19 Epidemic. A cross-sectional survey. Int J Environ Res Public Health 2020; 17(14): 5218-20. 